\title{
ORIGINAL RESEARCH \\ High-Resolution In Utero 3D MR Imaging of Inner Ear Microstructures in Fetal Sheep
}

\author{
J.-H. Buhk \\ M. Frisch \\ J. Yamamura \\ J. Graessner \\ G. Adam \\ U. Wedegärtner
}

\begin{abstract}
BACKGROUND AND PURPOSE: Developmental inner ear abnormalities can occur due to embryopathies as well as in the context of syndromal diseases like the CHARGE association. In severe cases, an early and definite in utero diagnosis is important for decision-making; here, fetal MR imaging can be a helpful tool. We present results of performing high-resolution MR imaging of the inner ear structures of fetal sheep in vivo.
\end{abstract}

METHODS AND MATERIALS: Six ewes carrying singleton fetuses (mean gestational age, 120 days) were examined under general anesthesia at 1.5T. A 3D true FISP sequence with isotropic voxel size $(0.7 \mathrm{~mm}$ ) was applied; acquisition time was 2:35 minutes. For a standard of reference, 1 stillborn lamb of equivalent gestation age was examined. Image analysis was performed in consensus by 2 radiologists regarding the depiction of anatomic landmarks on a 5-point scale. Motion artifacts were quantified on a 3-point scale.

\begin{abstract}
RESULTS: The turns and modiolus of the cochlea as well as the origins of all 3 semicircular canals of the vestibular system of both sides could be reliably identified in every animal. Motion artifacts due to maternal breathing excursions or movements of the fetus were minimal. In case of breech presentation, the ventilation of the ewe had to be paused during the image acquisition to achieve acceptable results.
\end{abstract}

CONCLUSIONS: High-resolution intrauterine MR imaging of the inner ear microstructures in an animal model is feasible. However, the acquisition time of the sequence applied is still too long to perform such measurement in a clinical setting.
$\mathbf{F}_{\mathrm{n}}$ tal MR imaging is a reasonable and increasingly accepted method in addition to fetal sonography for diagnostic imaging of both developmental and acquired fetal disorders; in critical cases, fetal MR imaging can serve as the basis for important clinical decisions. ${ }^{1-10}$ Today, virtually all fetal organ systems can be examined with MR imaging in high quality, as well as surrounding structures like the placenta. ${ }^{10}$ Moreover, functional fetal MR imaging can be performed (eg, for gross fetal movements, cardiac function, brain oxygenation, tractography, and spectroscopy). ${ }^{10-14}$

Inner ear malformations can occur in the context of several acquired embryopathies such as the following: infections (rubella, toxoplasma, lues), teratogenous medication (thalidomide), ionizing radiation, and hypoxia. ${ }^{15}$ These can occur monosymptomatically or as a part of a syndromal diseasefor example, Pendred, Franceschetti, Goldenhar, Alport, Usher, Waardenburg, Klippel-Feil, Norrie, Wildervanck, Jervell-Lange-Nielsen, and Down syndromes as well as the CHARGE association. ${ }^{15-17}$ In humans, the cochlear duct develops in an earlier period (until the eighth week) than the saccule, utricle, and endolymphatic ducts (completed at the

Received January 25, 2011; accepted after revision April 13

From the Department of Diagnostic and Interventional Radiology (J.-H.B., M.F., J.Y., G.A., U.W.), University Medical Center Hamburg Eppendorf, Hamburg, Germany; and Siemens Healthcare (J.G.), Hamburg, Germany.

Please address correspondence to Jan-Hendrik Buhk MD, Department of Diagnostic and Interventional Radiology, University Medical Center Hamburg Eppendorf, Martinistr, D-20246 Hamburg, Germany; e-mail: jbuhk@uke.de

http://dx.doi.org/10.3174/ajnr.A2736 11th week); the semicircular canals are complete even later (between the 19th and 22nd weeks). Therefore, a problem occurring early (between the third and fifth weeks of gestation) can result in a complete "common cavity" inner ear malformation; after the eighth week, it can result in malformation of the saccule, utricle, and the semicircular canals while the cochlea is normally developed. ${ }^{18}$

In most of the aforementioned diseases, the diagnosis can be confirmed by other modalities apart from MR imaging. However, if the diagnosis of a syndromal disease with an unknown genetic defect is in question and an inner ear malformation is a part of the diagnostic pattern, a robust method for intrauterine imaging of the inner ear microstructures would be desirable. ${ }^{17,18}$

Inspired by the work of Tilea et al, ${ }^{17}$ who presented 2 cases in which the diagnosis of the fetal malformations eventually was based on the findings of the fetal inner ear, we present our experimental series regarding targeted MR imaging of the inner ear structures in fetal sheep in vivo. The pregnant sheep model is established for assessment of congenital abnormalities by MR imaging; however, there currently is no study presenting normal findings regarding the fetal sheep's inner ear microstructures or their embryology. ${ }^{19}$ A study on the morphology of human and sheep inner ear structures on CT scans revealed a high grade of comparability; therefore, we can assume a significant degree of similarity even regarding the steps of development. ${ }^{20}$

The adequate positioning of the usually applied fast 2D sequences in fetal MR imaging can be very time-consuming 


\begin{tabular}{|c|c|c|c|c|c|c|c|}
\hline \multicolumn{8}{|c|}{ Scores regarding the identification of the anatomic structures and of the assessment of the artifacts load ${ }^{\text {a }}$} \\
\hline Animal & Cochlea & Utriculus & Lateral Canal & Anterior Canal & Posterior Canal & $\mathrm{IAM}^{\mathrm{b}}$ & Artifacts \\
\hline A & 4 & 4 & 1 & 2 & 4 & 3 & Moderate \\
\hline B & 4 & 4 & 1 & 1 & 4 & 4 & Unremarkable \\
\hline C & 5 & 5 & 2 & 1 & 4 & 5 & Unremarkable \\
\hline D & 3 & 3 & 1 & 1 & 1 & 3 & Severe \\
\hline$E$ & 4 & 4 & 1 & 1 & 3 & 3 & Moderate \\
\hline $\mathrm{F}$ & 5 & 5 & 1 & 1 & 4 & 4 & Moderate \\
\hline Ref. & 5 & 5 & 3 & 3 & 5 & 3 & Unremarkable \\
\hline
\end{tabular}

Note:- -Ref. indicates reference; 1, not visible; 2, visible, not diagnostic; 3, moderate but sufficient; 4, good quality; 5, excellent quality.

a First, the depictability of the lateral and anterior semicircular canals is also moderate in the reference examination and, therefore, likely to be a normal anatomic finding at that stage of development. Second, severe artifacts only occurred in animal $D$ due to breech presentation of the fetus.

${ }^{b}$ Scoring includes visibility of cranial nerves VII and VIII.

and especially for inner ear imaging; orthogonal and anatomically correct section orientation is crucial. Therefore, this study features a dedicated 3D MR sequence for imaging the fetal inner ear in sheep, generating isotropic voxels to allow multidirectional postprocessing after the acquisition, resulting in MPRs in an orthogonal direction.

The purposes of this study were to demonstrate the feasibility of high-resolution MR imaging of the fetal inner ear structures in vivo in a large-animal model and to open a possible clinical perspective of 3D fetal MR imaging.

\section{Materials and Methods}

All experimental protocols were reviewed, and the local animal protection authorities approved the usage of ewes and fetuses.

The fetal MR imaging measurements were performed on 6 pregnant ewes carrying singleton fetuses with a mean gestational age of 120 days. Pregnancy in sheep lasts 150 days; that time point, therefore, corresponds to the 32nd week of gestation in humans.

For MR imaging measurements, the ewes were sedated with $20 \mathrm{mg}$ of diazepam and $250 \mathrm{mg}$ of ketamine administered intravenously in the external jugular vein and repeated as required. The ewes were then intubated and transferred from the animal care facility to the MR imaging unit. During the MR imaging examinations, the ewes were artificially ventilated (1\%-2\% isoflurane in oxygen/nitrous oxide; tidal volume, $600-1000 \mathrm{~mL} ; 6-11$ cycles per minute). For monitoring, the maternal oxyhemoglobin saturation was sampled with a Pulse-Oximeter system connected to the maternal tongue (Nonin Medical, Plymouth, Minnesota). The ewe was connected to the respirator, which was situated outside the MR imaging scanner room, with standard anesthesia hoses. All animals were examined in left lateral position.

\section{MR Imaging of the Fetal Inner Ear}

MR imaging was performed at 1.5T (Magnetom Symphony; Siemens, Erlangen, Germany), applying the standard clinical surface coil (4channel phased array). For imaging of the fetal inner ear, a highly T2-weighted 3D true FISP sequence was applied with the following parameters: TR, $4.3 \mathrm{~ms}$; TE, $1.85 \mathrm{~ms}$; flip angle, $40^{\circ}$; isotropic voxel size, 0.7 -mm edge length; acquisition time, 2:35 minutes. The stack consisted of 64 sections, which resulted in head-feet volume coverage of $4.5 \mathrm{~cm}$. A symmetric orientation according to the inner ear anatomy was not mandatory due to the isotropic character. No parallel imaging was applied to avoid signal-intensity degradation. 3D true FISP as a balanced steady-state free precession sequence was chosen over 3D CISS because it offers the highest signal intensity-to-noise ratio with time. ${ }^{21}$

\section{Standard of Reference}

To get the best possible anatomic reference, we examined, shortly after death (6 hours), 1 lamb that was prematurely stillborn (142nd day). The stillborn lamb was examined on the same hardware with a high-resolution 3D CISS sequence with the following parameters: TR, $14 \mathrm{~ms}$; TE, $7 \mathrm{~ms}$; flip angle, $70^{\circ}$; isotropic voxel size, 0.4 -mm edge length; acquisition time, 10 minutes, 32 seconds.

The 3D true FISP sequence applied in utero could not be successfully performed in the stillborn lamb due to gross image distortion and loss of T2 contrast, likely because of air-filled surrounding tissues and a shift of the T2/T1 ratio in postmortem tissues.

\section{Image Processing and Evaluation}

Postprocessing of the image data into axial and coronal/paracoronal MPRs and image viewing were performed by using OsiriX Imaging Software (http://www.osirix-viewer.com); both average and MIPs were used. ${ }^{22}$

For the evaluation of image quality, the visualization of anatomic structures and the presence of motion artifacts were assessed. Two experienced radiologists (J.-H.B., M.F.; cumulative experience, 11 years) performed a consensus reading regarding the reliable identification of the following important anatomic landmarks: cochlea, utriculus, semicircular canals, and IAM with the seventh and eighth cranial nerve. For the rating of the diagnostic quality, we applied a 5-point scale: $1=$ not visible; $2=$ visible, not diagnostic; $3=$ moderate but sufficient; $4=$ good quality; $5=$ excellent quality. Artifacts were quantified on the basis of a 3 -point scale; $1=$ severe, $2=$ moderate, $3=$ unremarkable.

\section{Results}

It was possible to perform MR imaging of the inner ear in all 6 fetuses. An overview of the scoring results is provided in the Table.

\section{Motion Artifacts}

On average, motion artifacts were rated moderate. Artifacts can be generated by fetal as well by maternal movements. As shown in the Table, there was only 1 animal with severe artifacts due to maternal breathing (animal D, fetus in breech presentation). In this case, the ventilation of the ewe was paused for 2 minutes, 30 seconds during the image acquisition to achieve at least acceptable results, but stomach movements were still present. In 3 animals, motion artifacts were moderate. In 2 animals, no motion artifacts were detected, similar to the reference scan. 

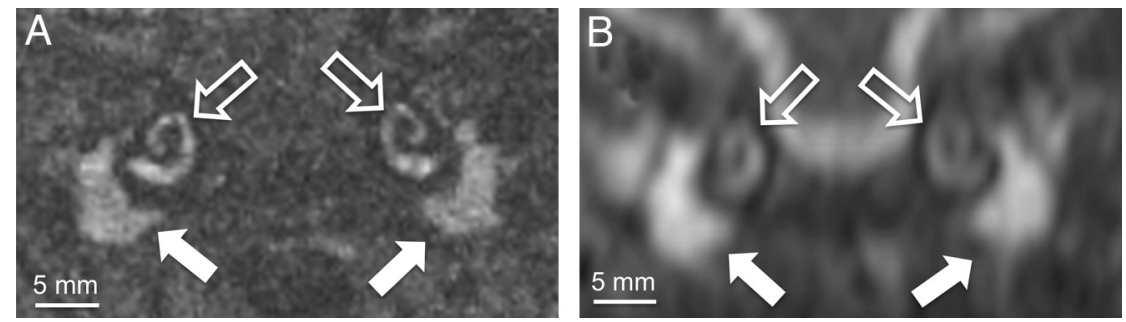

Fig 1. Coronal view (thin-section MIP; section thickness, $0.5 \mathrm{~mm}$ ) shows the normally developed cochlea (open arrows) and the tympanic cavity (closed arrows), which are both (normal findings in utero) fluid-filled in the reference examination ( $A, 3 \mathrm{D}$ CISS, stillborn lamb) as well as in vivo (B, 3D True FISP).
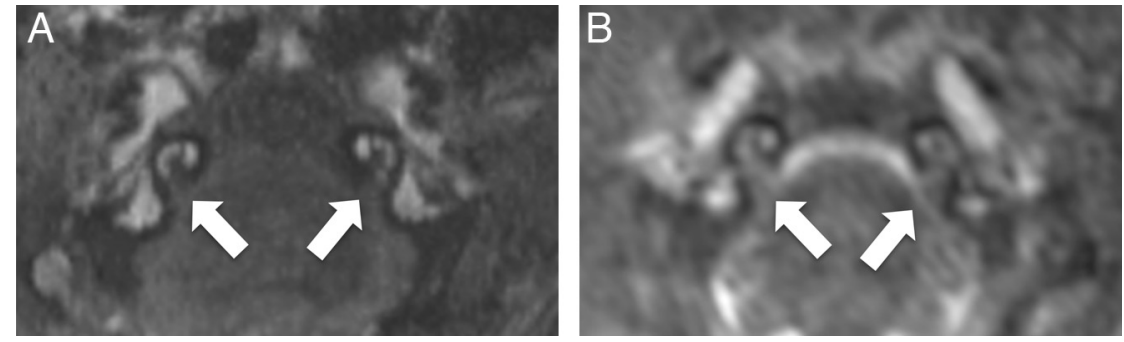

Fig 2. This set of axial views of the reference examination ( $A, 3 \mathrm{D}$ CISS, stillborn lamb) and in vivo ( $B, 3 \mathrm{D}$ True FISP; both thin-section MIPS; section thickness, $0.7 \mathrm{~mm}$ ) displays the IAM with the seventh and eighth cranial nerve (arrows) and a cross-section through the normally developed cochlear modiolus, which is also well-depicted.
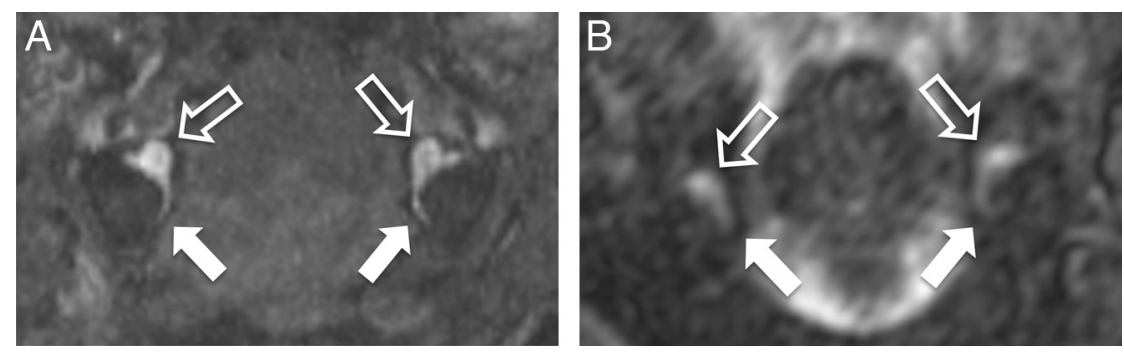

Fig 3. The axial views of the reference examination ( $A$, 3D CISS, stillborn lamb) and in vivo ( $B, 3 \mathrm{D}$ True FISP; both thin-section MIPs; section thickness, $2.0 \mathrm{~mm}$ ) display the utriculus (open arrows) and the medial section of the posterior semicircular canal (closed arrows). The utricular ostium of the lateral semicircular canal can be seen as well in both images.

\section{Identification of Anatomic Landmarks}

On average, the cochlea, utriculus, and the IAM were rated as having good image quality. The identification of the posterior semicircular canal of the vestibular system was moderate but sufficient, whereas the anterior and lateral semicircular canals were not visible (Table).

The basal turns of the cochlea are displayed very well by using a coronal view with a good correlation to the reference animal (Fig 1); axial views provide the best visualization of the modiolus as shown in Fig 2.

The origins of all 3 semicircular canals of the vestibular system of both sides can be identified in every fetus (Fig 3). Only the posterior semicircular canal is completely visible as a fluid-filled structure. This finding correlates with the finding in the reference animal, in which the lateral and the anterior semicircular canals were not completely visible as well (Fig 3).

The normally configured IAM is relatively easily depicted on axial MPRs of the in vivo data as shown in Fig 2. The contained cranial nerves VII and VIII present as faint hypointense linear structures (Fig 2). A further differentiation into the vestibular, cochlear, and facial segments was not possible. Because the normal T2 hyperintensity of the CSF was already lost in the reference animal, the contrast regarding the IAM is not optimal (Fig 2).

\section{Discussion}

Imaging the petrous bone and especially the inner ear microstructures in adolescents or adults is a standard examination in today's MR imaging or CT. Among the main reasons for performing MR imaging of the inner ear in children may be developmental abnormalities, which are either due to embryopathies or occur in the context of syndromal diseases like the CHARGE association or ANSD. ${ }^{23}$ Early diagnosis of morphologic abnormalities is very important and helpful for decisionmaking, (eg, when considering a CI). However, with regard to CIs, there already are potent electrophysiologic methods to diagnose hearing impairment and/or loss in infants early, though this does not exclude the need for proper imaging in some cases. Because fetal MR imaging today is also often performed when an embryopathy or syndrome is in question, it is reasonable in these cases to have a closer look at the inner ear as well.

This is the first animal study on dedicated 3D MR imaging of the fetal inner ear microstructures, to our knowledge. We are aware that the sequence used is not yet perfectly suitable for transferring to standard clinical human fetal MR imaging protocols because the acquisition time ( $>2$ minutes) is still very long for that situation. Even with the patient under mild sedation, we would not be able to definitely eliminate some 
fetal movements during the measurement. In our experimental setting, the examinations were performed with the subject under general anesthesia, which is not an ethically acceptable condition for performing human fetal MR imaging.

However, under experimental conditions, high-resolution 3D MR imaging of the relevant fetal inner ear microstructures is feasible. There were only minor drawbacks due to the case of breech presentation of 1 fetus, which led to impairments through maternal breathing and stomach movements.

The normally developed cochlea, as the most recognizable and important inner ear structure, was well visualized in all fetuses as well as in our reference animal. Because the fluid-filling of the semicircular canals occurs incompletely in vivo in humans as well as in the reference animal, that may be a normal finding at that state in the development of fetal sheep. This finding can be considered in line with the relatively late completion of the semicircular canals in human embryology. ${ }^{18}$ However, the origins of the canals in the utriculus can be visualized in all cases apart from the 1 in breech presentation, indicating normal development of the semicircular canals in all animals. That finding is important because in some reported cases of CHARGE syndrome, there was only deformation or absence of the semicircular canals. ${ }^{23}$ The IAM with the included cranial nerves VII and VIII was also displayed in diagnostic quality, which is an important finding because hypoplasia or atresia can occur in several cases of ANSD. ${ }^{23}$

The main advantage of applying a 3D sequence with isotropic resolution for inner ear imaging is the possibility of postprocessing MPRs. A substantial amount of time can be saved by not having to arrange the sequence stack in an anatomically correct orientation. On the other hand, in our study, this gain in time was neutralized by the duration of the sequence tested. With regard to good clinical practice, an even faster 3D sequence would desirable. On the other hand, a 2D sequence with ultrahigh in-plane resolution could serve as an alternative.

In addition to the developments of functional fetal MR imaging during the past years, we here provide a new approach to morphologic fetal MR imaging in an animal model, which may, in the future, serve as an extension to a modern fetal MR imaging work-up. ${ }^{10-14}$ We would be glad to perform a follow-up study with an improved sequence suitable for the human setting, to look for possible clinical implications.
Disclosures: Joachim Graessner, Research Support (including provision of equipment or materials). Siemens Healthcare, Details: employee; Ownership Interest. Siemens, Details: stockholder.

\section{References}

1. Levine D, Barnes PD, Madsen JR, et al. Central nervous system abnormalities assessed with prenatal magnetic resonance imaging. Obstet Gynecol 1999;94:1011-19

2. Levine $\mathrm{D}$, Barnes PD. Cortical maturation in normal and abnormal fetuses as assessed with prenatal MR imaging. Radiology 1999;210:751-58

3. Simon EM, Goldstein RB, Coakley FV, et al. Fast MR imaging of fetal CNS anomalies in utero. AJNR Am J Neuroradiol 2000;21:1688-98

4. Levine D, Barnes PD, Robertson RR, et al. Fast MR imaging of fetal central nervous system abnormalities. Radiology 2003;229:51-61

5. Frates MC, Kumar AJ, Benson CB, et al. Fetal anomalies: comparison of MR imaging and US for diagnosis. Radiology 2004;232:398-404

6. Prayer D, Brugger PC, Prayer L. Fetal MRI: techniques and protocols. Pediatr Radiol 2004;34:685-93

7. Gressens P, Luton D. Fetal MRI: obstetrical and neurological perspectives. Pediatr Radiol 2004;34:682-84

8. Glenn OA, Barkovich J. Magnetic resonance imaging of the fetal brain and spine: an increasingly important tool in prenatal diagnosis-part 2. AJNR Am J Neuroradiol 2006;27:1807-14

9. Glenn OA, Barkovich AJ. Magnetic resonance imaging of the fetal brain and spine: an increasingly important tool in prenatal diagnosis-part 1. AJNR Am J Neuroradiol 2006;27:1604-11

10. Chung R, Kasprian G, Brugger PC, et al. The current state and future of fetal imaging. Clin Perinatol 2009;36:685-99

11. Wedegartner U, Tchirikov M, Schafer S, et al. Functional MR imaging: comparison of BOLD signal intensity changes in fetal organs with fetal and maternal oxyhemoglobin saturation during hypoxia in sheep. Radiology 2006;238:872-80

12. Kasprian G, Brugger PC, Weber M, et al. In utero tractography of fetal white matter development. Neuroimage 2008;43:213-24

13. Pugash D, Krssak M, Kulemann V, et al. Magnetic resonance spectroscopy of the fetal brain. Prenat Diagn 2009;29:434-41

14. Yamamura J, Schnackenburg B, Kooijmann H, et al. High resolution MR imaging of the fetal heart with cardiac triggering: a feasibility study in the sheep fetus. Eur Radiol 2009;19:2383-90

15. Gottschalk S, Gehrking E, Petersen D. Fehlbildungen des Felsenbeins. Clin Neuroradiol 2006; 16:17-32

16. Pagon RA, Graham JM, Zonana J, et al. Coloboma, congenital heart disease, and choanal atresia with multiple anomalies: CHARGE association. J Pediatr 1981;99:223-27

17. Tilea B, Garel C, Menez F, et al. Contribution of fetal MRI to the diagnosis of inner ear abnormalities: report of two cases. Pediatr Radiol 2006;36:149-54

18. Casselman JW, Offeciers EF, De Foer B, et al. CT and MR imaging of congential abnormalities of the inner ear and internal auditory canal. Eur J Radiol 2001;40:94-104

19. Ward VL, Estroff JA, Nguyen HT, et al. Fetal sheep development on ultrasound and magnetic resonance imaging: a standard for the in utero assessment of models of congenital abnormalities. Fetal Diagn Ther 2006;21:444-57

20. Seibel VA, Lavinsky L, Irion K. CT scan: sheep and human inner ear morphometric comparison. Braz J Otorhinolaryngol 2006;72:370-76

21. Scheffler K, Lehnhardt S. Principles and applications of balanced SSFP techniques. Eur Radiol 2003;13:2409-18

22. Rosset A, Spadola L, Ratib O. OsiriX: an open-source software for navigating in multidimensional DICOM images. J Digit Imaging 2004;17:205-16

23. Huang BY, Roche JP, Buchman CA, et al. Brain stem and inner ear abnormalities in children with auditory neuropathy spectrum disorder and cochlear nerve deficiency. AJNR Am J Neuroradiol 2010;31:1972-79 